Kalinowsky · Kampfplatz Justiz 
Studien und Materialien zum Rechtsextremismus herausgegeben von Prof. Dr. Eike Hennig

Band 3 


\section{Kampfplatz Justiz}

\section{Politische Justiz \\ und Rechtsextremismus in der \\ Bundesrepublik Deutschland \\ $1949-1990$}

Harry H. Kalinowsky

Centaurus Verlag \& Media UG 1993 
Umschlagfoto: Ullstein - dpa

Zum Autor: Dr. Harry H. Kalinowsky studierte Politische Wissenschaft, Soziologie und Völkerrecht an der Universität Bonn, Kriminologie an der Universität Hamburg und ist zur Zeit als Dozent an der Zivildienstschule Buchholz tätig.

Veröffentlichung: Rechtsextremismus und Strafrechtspflege, Bonn 1986.

Die Deutsche Bibliothek - CIP-Einheitsaufnahme

Kalinowsky, Harry H.:

Kampfplatz Justiz : politische Justiz und Rechtsextremismus in der Bundesrepublik Deutschland 1949 - 1990 / Harry Hugo

Kalinowsky.-Pfaffenweiler : Centaurus-Verl.-Ges., 1993

(Studien und Materialien zum Rechtsextremismus ; Bd. 3)

Zugl.: Duisburg, Univ., Diss., 1993

ISBN 978-3-89085-879-1

ISBN 978-3-86226-425-4 (eBook)

DOI $10.1007 / 978-3-86226-425-4$

NE: GT

ISSN 0940-2977

Alle Rechte, insbesondere das Recht der Vervielfältigung und Verbreitung sowie der Übersetzung, vorbehalten. Kein Teil des Werkes darf in irgendeiner Form (durch Fotokopie, Mikrofilm oder ein anderes Verfahren) ohne schriftliche Genehmigung des Verlages reproduziert oder unter Verwendung elektronischer Systeme verarbeitet, vervielfältigt oder verbreitet werden.

(C) CENTAURUS-Verlagsgesellschaft mit beschränkter Haftung, Pfaffenweiler 1993

Satz: Vorlage des Autors 
Wissenschaftler haben nicht oft die Gelegenheit, empirische Arbeiten aktualisieren und fortschreiben zu können. Dank der Unterstützung des Bundesministers der Justiz wurde mir die Gelegenheit gegeben, die empirische Untersuchung $z u$ Strafverfahren wegen mutmaßlicher rechtsextremistischer Aktivitäten und Erscheinungen mit der Einbeziehung neuer Fälle und der Erweiterung des zeitraums der Untersuchung fortschreiben $z u$ können. Mit der Fortsetzung der empirischen Untersuchung konnte ich auch meinen eigenen Forschungsstandpunkt überdenken und erfahren, daß Forschung immer auch ein Entwicklungsproze $\beta$ für den Wissenschaftler selbst ist. Die Aktualisierung der Studie nutzte ich daher zu einer Weiterentwicklung der Forschungsperspektive. Die Dissertation versucht, den Begriff Politische Justiz analytisch sinnvoll zu gestalten, um all die Prozesse in den Blick nehmen $z u$ können, die Justiz zu einer politischen Justiz werden lassen können. Die Entwicklung der Justiz und des Rechtsextremismus werden in der nächsten zeit vermutlich $A n l a ß z u$ einer intensiven Diskussion geben. Mit der vorliegenden Arbeit wird aufgezeigt, wie sich in den letzten vierzig Jahren Justiz und Rechtsextremismus aufeinander bezogen haben und welche Faktoren und Mechanismen hier wirksam wurden. Insoweit hoffe ich mit dieser Arbeit dazu beizutragen, daß den Herausforderungen und Problemen in diesem spezifischen Beziehungsgefüge die entsprechende Aufmerksamkeit geschenkt wird.

Vorworte dienen auch dem Dank. Mein Dank gilt an erster Stelle Herrn Prof. Dr. Heribert Schatz, der mich mit Geduld und Unterstützung durch den Erstellungsprozeß dieser Arbeit begleitet hat. Weiterhin gilt mein Dank Herrn Dr. Dr. jur. Konrad Hobe, der es mir überhaupt ermöglicht hat, die empirische Arbeit durchführen zu können.

Meine Frau und meine Kinder Katharina, Kilian und Klara wissen, daß ich ihre Geduld in besonderem Maße strapazieren mußte. Ihnen sei zum Dank meine Arbeit gewidmet. 
Vorwort

Inhaltsverzeichnis

Tabellenverzeichnis

Grafikverzeichnis

Abkürzungen

... vii

$\ldots x i$

$\cdots \quad x v$

... xvii

1 .

2 .

2.1

2.1.1

2.1 .2

2.1 .3

2.1 .4

2.1 .4 .1

2.1 .4 .2

2.2

2.3

2.4

2.5

2.6

3.

3.1 .

3.2

3.3

3.4

3.5

3.5 .1

3.5 .2

3.5 .2 .1

3.5 .2 .2

3.5 .2 .3

3.5 .2 .4

3.5 .2 .5

3.5 .2 .6

$3 \cdot 5 \cdot 3$

$3.5 \cdot 3.1$

3.5 .3 .2

3.5 .4

3.5 .4 .1

3.5 .4 .2

3.5 .4 .3

3.5 .5

3.6

3.6 .1

3.6 .2

\section{Einleitung}

Bezugsrahmen

Politische Justiz

Begriffsproblematik

Basisdefinition Politische Justiz

Kritische würdigung

Konstituierungsmodi Politischer Justiz

Definitionsmacht als Begriffskriterium

Politikbegriff als Kriterium

Politische Kriminalität

Gerichtsarena

Politische Kultur

Rechtsextremismus

Konfiguration des Bezugsrahmens

Historische Analyse

Politisch-gesellschaftliche Rahmenbe-

dingungen der Bundesrepublik Deutschland

1949 - 1990

Politische Kultur der Mehrheits-

gesellschaft

Organisations- und Aktionsentwicklung im Rechtsextremismus

Politische Subkultur Rechtsextremismus

Gerichtsarena

Aspekte der Nichtnutzung

Gerichtsarena der fünfziger Jahre

Parteiverbot der SRP

Pathologisierung

Skandalisierung und Diskreditierung

Kommunikationsgrenzen, Teil 1

Kommunikationsgrenzen, Teil 2

Interaktionsgrenzen

Gerichtsarena in den sechziger Jahren

Legitimationsdruck

Formierungsdruck

Gerichtsarena seit den siebziger Jahren

Übergangsphänomene

Agitatoren und Terroristen

Skins und Revisionisten

Justizkritik

Normkonstituierte politische Justiz

Stunde Null des politischen Strafrechts

Strafrecht als waffe gegen den Rechts-

extremismus
... 1

... 11

$\ldots 13$

... 14

... 20

... 22

... 27

... 30

$\ldots 32$

... 37

$\ldots 43$

... 50

... 54

... 61

... 64

... 65

... 70

... 80

.. 109

... 124

... 124

... 131

... 132

... 135

... 137

... 138

... 139

... 144

... 147

... 147

... 150

... 153

... 153

... 158

... 177

... 181

$\ldots 186$

... 190

... 199 


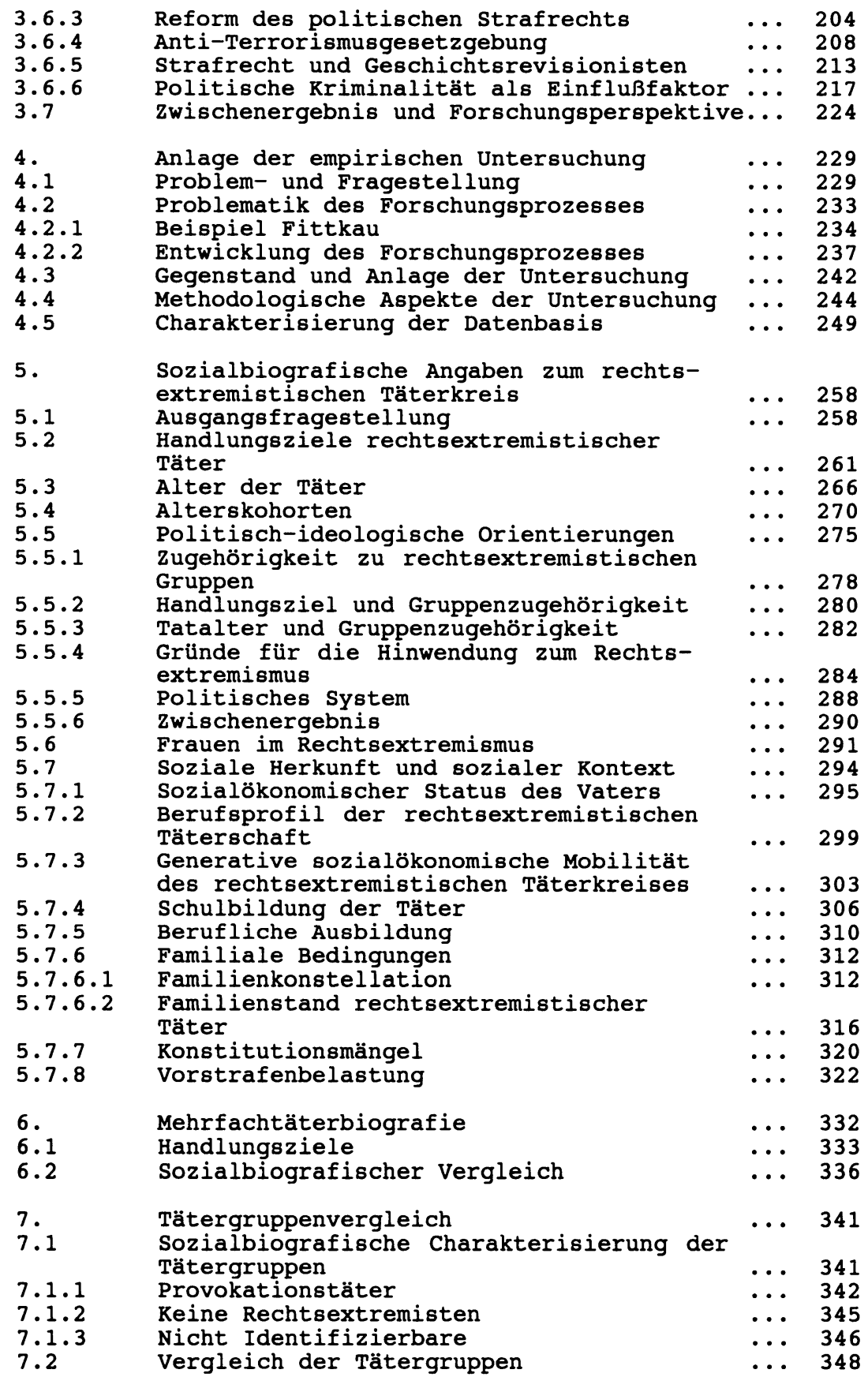


7.3 Rechtsextremisten und Nicht Identifizierbare

8.

8.1

Handlungsziele aller Täter

... 354

-.. 355

8.2

Rechtsextremistische Straftaten

... 360

Rechtsextremistisches Deliktprofil

... 361

Populationsspezifisches Deliktprofil

... 369

8.3

Besondere Strafvorschriften gegen den

Rechtseextremismus

8.3.1

S 86 StGB

8.3 .2

S $86 a$ StGB

8.3.3

8.4

Sonstige einschlägige Vorschriften

... 373

... 373

... 375

... 378

Verurteilungen nach ausgewählten $\mathrm{Be}-$ stimmungen

8.5

Aussagetendenzen in der Agitationskriminalität

... 382

... 390

9.

9.1

Aspekte der Straf- und Gerichtsverfahren wegen mutmaßlicher rechtsextremistischer Aktivitäten und Erscheinungen

Mit Strafverfahren wegen mutmaßlicher rechtsextremistische Aktivitäten befaßte

Gerichte

9.1 .1

Gericht

-

9.1 .2

9.1 .3

... 396

... 397

Regionales Belastungsprofil der Justiz $\quad . .399$

Länge der Gerichtsverhandlungen in

der ersten Instanz

... 403

9.1 .4

Verhandlungsdauer in der ersten Instanz

... 408

9.1 .5

zeit zwischen erstinstanzlichem Urteil

und Rechtskraft

9.1 .6

Zeit zwischen Anklageerhebung und Rechts-

kraft des Urteils

9.2

Rechtsmittelverfahren

9.2 .1

Häufigkeit und Erfolg der Berufung

Häufigkeit und Erfolg der Revision

‥ 409

9.2 .2

9.3

Vergleich der strafvorschriften in der

Anklageschrift und im rechtskräftigen

Urteil

9.4

Aspekte der Untersuchungshaft

‥ 425

... 438

10. Strafzumessung und Ausschöpfung des

Strafrahmens

10.1

Aburteilungen und Sanktionen

10.2

Sanktionspolitik

10.2 .1

Sanktionsprofil

Täter

... 412

... 413

... 415

... 419

10.2.2 Sanktionen nach erstinstanzlichem Gericht und Gerichtsort

10.2.2.1 Erstinstanzliches Gericht und Sanktionsprofil

$10 \cdot 2 \cdot 2 \cdot 2$

pänd

$10 \cdot 2 \cdot 3$

10.3

Untersuchungshaft und Sanktionsprofil

... 444

... 445

-.. 449

Strafzumessung und Ausschöpfung des

Strafrahmens

... 449

$10 \cdot 3 \cdot 1$

Strafzumessung und Ausschöpfung des

Strafrahmens bei der Freiheitsstrafe

$10 \cdot 3 \cdot 1 \cdot 1$

Höhe der Freiheitsstrafe

‥ 452

... 452

... 454

... 459

... 461

... 465

... 465 
10.3.1.2 Ausschöpfung des Strafrahmens bei der Freiheitsstrafe

... 467

10.3.2 Strafzumessung und Ausschöpfung des Strafrahmens bei der Jugendstrafe

.. 479

10.3.3 Strafzumessung und Ausschöpfung des

10.3.4 Strafrahmens bei der Geldstrafe
Vergleich der Ausschöpfungsanteile

... 484

-.. 487

11.

11.1

11.2

11.2 .1

Zusammenfassung der empirischen Analyse

... 490

Bezugsrahmen

Täter

... 490

Keine Rechtsextremisten

$11 \cdot 2 \cdot 2$

Rechtsextremistische Straftäter

Taten

11.3

Strafverfahren

... 492

... 493

... 498

... 504

... 506

11.5

Sanktionen

... 510

12 .

12.1

12.2

12.3

12.4

12.5

13 .

13.1

13.2
Integrative Zusammenschau der Ergebnisse Politische Justiz

Gerichtsarena

Politische Kriminalität

Rechtsextremismus

Politische Kultur
... 514

... 515

... 519

... 523

... 525

... 528

Politische Justiz gegen rechts im vereinten Deutschland _.. 531 Gefahrenmomente der politischen Justiz $\quad . .5531$ Politische Justiz im vereinten Deutschland... 535

Literaturverzeichnis

... 541

Quellenverzeichnis

... 570 
Tabelle

Nummer

301

302

303

304

401

402

403

404

501

502

503

504

505

506

507

508

509

510

511

512

513

514

515

516

517

518

519

520

521

522
Bezeichnung

Wahlanteile führender rechtsextremer

Parteien 1949 - 1990

Typologie der Aggregatzustände

Entwicklungsphasen des organisierten

Rechtsextremismus 1945 - 1990

Gesetzesverstöße 1990 durch Extremisten

Beschreibung der Grundgesamtheit

Tätergruppenvergleich

Gerichtsverfahren pro Rechtsextremist

Verfahrensübersicht nach Jahr der

Rechtskraft und Datensatzzugehörigkeit

Handlungsziele rechtsextremistischer

Täter

Alter der Täter zur zeit der Tat

(Vergleich 1. und 2. Untersuchung, alt neu)

Alter zur zeit der Tat und Handlungsziel

Alterskohorten der Rechtsextremisten (RE)

Jahrgangskohorten und Handlungsziel

Zugehörigkeit $\mathrm{zu}$ rechtsextremistischen

Gruppen nach ideologischen Bereichen

Handlungsziel und Gruppenzugehörigkeit

Alter zur zeit der Tat und Gruppenzuge-

hörigkeit

Hinwendung zum Rechtsextremismus

Handlungsziel und Hinwendungsgrund

Handlungsziel und Sozialisationshintergrund Politisches System

Alter zur zeit der Tat bei den weiblichen

Rechtsextremisten (RE), Vergleich in of mit

1. Untersuchung

Verurteilte Rechtsextremisten nach

Geschlecht und Handlungsziel

Beruf des Vaters

296

Sozialökonomischer Status des Vaters und

298

Handlungsziel (SöS=Sozialökonomischer Status)

Beruf des Täters zur zeit der Tat

300

Sozialökonomischer Status der rechtsex-

tremistischen Täter zur zeit der Tat und

Handlungsziel, absolute Werte

Sozialökonomischer Status der rechtsex-

tremistischen Täter zur Zeit der Tat und

Handlungsziel, Prozentwerte

Beruf des Täters und Beruf des Vaters

(Sozialökonomischer Status)

Schulbildung der rechtsextremistischen Täter 307

Schulbildung der rechtsextremistischen Täter 308 und Handlungsziel

Handlungsziel, wenn ein Abbruch von Aus-

bildungsverhältnissen festgestellt wurde 
Tabelle

Nummer

523

524

525

526

527

528

529

530

531

532

533

601

602

603

701

702

801

802

803

804

805

806
Bezeichnung

Seite

Familie als Sozialisationsinstanz

Familienkonstellation der rechtsextrem-

315

istischen Täter und Handlungsziel

Familienstand rechtsextremistischer Täter

im Alter von 20 bis 25 Jahren

Familienstand rechtsextremistischer Täter

insgesamt

Familienstand rechtsextremistischer Täter

320 und Handlungsziel

Handlungsziele bei den Tätern mit der Angabe 321 von Konstitutionsmängeln

Rechtsextremistische Täter mit Vorstrafen

nach dem Hintergrund bei den vorangegangenen Straftaten

Handlungsziele bei den Tätern mit Vorstrafen 325

Sonstige frühere Sanktionen gegen Rechtsex-

tremisten. Nach allgemeinem Strafrecht und

Jugendstrafrecht sowie Sanktionshintergrund

Schwerste frühere Sanktion bei rechtsex-

328

tremistischen Tätern

Schwerste frühere Sanktion und Handlungsziel 329 rechtsextremistischer Täter

Rechtsextremistischer Täterkreis nach

Verurteilungen und Aburteilungen

Handlungsziel nach Aburteilungen und Ver-

urteilungshäufigkeit bei den Rechtsex-

tremisten

Ausgewählte sozialbiografische Merkmale -

Vergleich der Täter nach Verurteilungs-

häufigkeit

Ausgewählte sozialbiografische Merkmale für

die nicht rechtsextremistischen Tätergruppen

Handlungsziele bei allen Verurteilungen

(1026)

Urteil - Rechtsextremisten nach Straftat-

feldern, schwerste Tat, 624 Täter

344

357

Schwerste Straftat von Rechtsextremisten,

364

geordnet nach Rechtsgütern

Verurteilungen - Straftatklassifikation nach
Tatfeldern, hier: Verurteilungen der Rechts-

extremisten insgesamt: 624 Täter, 748 Ver-

urteilungen

Verurteilungen - Straftatklassifikation nach

Tatfeldern, hier: Verurteilungen insgesamt=

901 Täter, 1026 Verurteilungen

Verurteilung nach \$ 86 StGB, Art der Tat-

handlung

Tathandlungen bei Verurteilungen nach 
Tabelle

Nummer

Bezeichnung

Seite

807

Art der Kennzeichen bei Verurteilung wegen

808

Verwendung/Verbreitung nach $\$ 86 a$ StGB

Verurteilungen nach ausgewählten Normen und

388

Normbereichen, unterschieden nach Tätergruppen

809

Hauptaussagetendenz der Propagandamittel/

Parolen bei Verstößen gegen die SS $86,86 \mathrm{a}$, 130,131 StGB

901

Gericht der ersten Instanz

398

902

903

Gericht der ersten Instanz bei Verfahren mit 402

rechtsextremistischem Bezug nach Bundes-

ländern

Hauptverhandlungstage (HV) erledigter

404

Verfahren 1986 insgesamt, Prozentwerte

904 Hauptverhandlungstage (HV) erledigter Ver-

405

fahren in der vorliegenden Untersuchung

905

Dauer der Hauptverhandlung erledigter Ver-

408

fahren in der vorliegenden Untersuchung in

Tagen

906

Zeit zwischen dem erstinstanzlichen Urteil

und der Rechtskraft des Urteils

907 Zeit zwischen Anklageerhebung und Rechts-

kraft des Urteils

908

Rechtsmittel im Utberblick

410

Rechtsmittelpolitik von Staatsanwaltschaft

und Verteidigung bei der Berufung

910 Berufungsentscheidung, gegliedert nach

Verteidigung und Staatsanwaltschaft

911 Rechtsmittelpolitik von Staatsanwaltschaft und Verteidigung bei der Revision

912 Revisionsentscheidung, gegliedert nach

Verteidigung und Staatsanwaltschaft

913 Rechtsmittelpolitik von Staatsanwaltschaft

und Verteidigung bei der zweiten Revision

914

Revisionsentscheidung bei der zweiten

414

416

Revision, gegliedert nach Verteidigung und

Staatsanwaltschaft

915

Anklage-Urteil-Vergleich, alle Verfahren der 429

Rechtsextremisten (794)

916 Anklage-Urteil-Vergleich, alle Verfahren 1382

419

420

423

424

917

Anordnung und Vollzug der Untersuchungshaft

Haftgrund bei den Verfahren insgesamt und

439

den Verfahren gegen Rechtsextremisten

919 Dauer der Untersuchungshaft

1001 Aburteilungen nach Sanktionsart und Verfahrensausgang insgesamt und Verurteilungsart für die Verfahren der Täter, die keine Rechtsextremisten sind und für die Rechtsextremisten ( $R E$ ) 
Tabelle

Nummer

Bezeichnung

Seite

1002

Sanktionen und Handlungsziele aller Straf-

450

täter

1003 Sanktion/Verfahrensausgang nach Gericht der ersten Instanz, alle Urteile

1004 Sanktion/Verfahrensausgang nach Bundes-

453

ländern für alle Aburteilungen

1005

Anordnung von Untersuchungshaft und späteres

455

Urteil

1006 Höhe der Freiheitsstrafe 465

1007

Aussetzung der Freiheitsstrafe zur Bewährung,467

1008

Vergleich mit Verurteiltenstatistik 1985

1009

des Strafrahmens bei der Freiheitsstrafe

Strafrahmen, Strafzumessung und Ausschöpfung 470

des Strafrahmens bei der Freiheitsstrafe für

die Rechtsextremisten

1010

Strafrahmen, Strafzumessung und Ausschöpfung 471

des Strafrahmens bei der Freiheitsstrafe für

die Rechtsextremisten mit mehr als einer

Aburteilung (nur für zeitige Freiheitsstrafe)

1011 Ausschöpfung des Strafrahmens nach dem

Umfang des Strafrahmens und bei der Ver-

urteilung nach nur einer strafvorschrift, bei

Idealkonkurrenz und bei Realkonkurrenz bei

der Freiheitsstrafe

1012 Ausschöpfung des Strafrahmens bei Frei-

heitsstrafe, straftatklassifikation nach

Tatfeldern

1013 Ausschöpfung des Strafrahmens bei Frei-

heitsstrafe nach Rechtsgitern

1014 Ausschöpfung des Strafrahmens nach Handzielen

$1015 \quad$ Nachträgliche Gesamtstrafenbildung mit 478

Freiheitsstrafe

1016

Höhe der bestimmten Jugendstrafe

1017

Strafrahmen, Strafzumessung und Ausschöpfung 481 des Strafrahmens bei der bestimmten Jugendstrafe

1018 Strafrahmen, Strafzumessung und Ausschöpfung des Strafrahmens bei der bestimmten Jugendstrafe für Rechtsextremisten, Durchschnittswerte

1019 Strafrahmen, Strafzumessung und Ausschöpfung 483 des Strafrahmens bei der bestimmten Jugendstrafe mit Strafaussetzung zur Bewährung

1020 Geldstrafe nach Strafrahmen, durchschnittliche Summe, durchschnittliche Höhe des Tagessatzes, durchschnittliche Anzahl der Tagessätze und durchschnittliche Ausschöpfung bei Rechtsextremisten und insgesamt 
Grafik

Nummer

3.1
3.2
3.3
3.4
3.5
3.6
3.7
3.8

4.1

4.2

5.1

5.2

5.3

5.4

5.5

5.6

5.7

5.8

5.9

5.10

5.11

5.12

5.13

5.14

6.1

6.2

6.3
Bezeichnung

Seite

Mitgliederentwicklung des organisierten

82

Rechtsextremismus

Verurteilungen mit Rechtskraft

152

Rechtsextremisten 1960 - 1969

Urteile pro Jahr (1970 - 1979)

Verurteilungen mit Rechtskraft

157

175

Rechtsextremisten 1980 - 1989

Staatsschutzdelikte $1954-1958$

218

219

221

223

Gesetzesverstöße 1965 - 1990

254

257

Untersuchung

Verfahren im Kernbereich nach Jahr der

Rechtskraft

Rechtsextremistische Handlungsziele

266

Altersgruppenvergleich Rechtsextremisten/ 269

Verurteilte 1986

Verteilung der Anzahl der verurteilten

273

Rechtsextremisten nach Geburtsjahrgang.

Jahrgangsverteilung Verurteilte

Gewaltziele und Kohorte. Prozentanteile pro

275

Kohorte

Mitgliedschaft nach Bereichen

279

Ideologiegruppe - Altersgruppe. Prozent- 283

vergleich

Hinwendungsgrund - Handlungsziel. Ideologie- 287

faktor Beziehungsfaktor

Altersgruppenverteilung Mann - Frau (RE)

Tatalter-Gruppen Geschlechtervergleich RE

Sozialer Kontext - Handlungsziel. Status

292

299

des Vaters und Gewaltziele

Schulbildung - Handlungsziel. Rechtsex- 309

tremismus

Familienstand RE. Prozentanteile

Handlungszielvergleich Täter mit Konstitutionsm./ RE Gesamt

318

321

326

Gesamt

Vergleich Handlungsziele nach vorheriger 330

Sanktion

Vergleich Handlungsziele. Mehr als $1 \mathrm{VU}-$

Insgesamt

Merkmalsvergleiche 1. Mehr als 1 VU - 1 VU 337

Merkmalsvergleiche 2. Mehr als 1 VU - 1 VU 337 

stand

9.1 Vergleich Hauptverhandlungstage Verfahren 407 


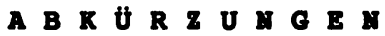

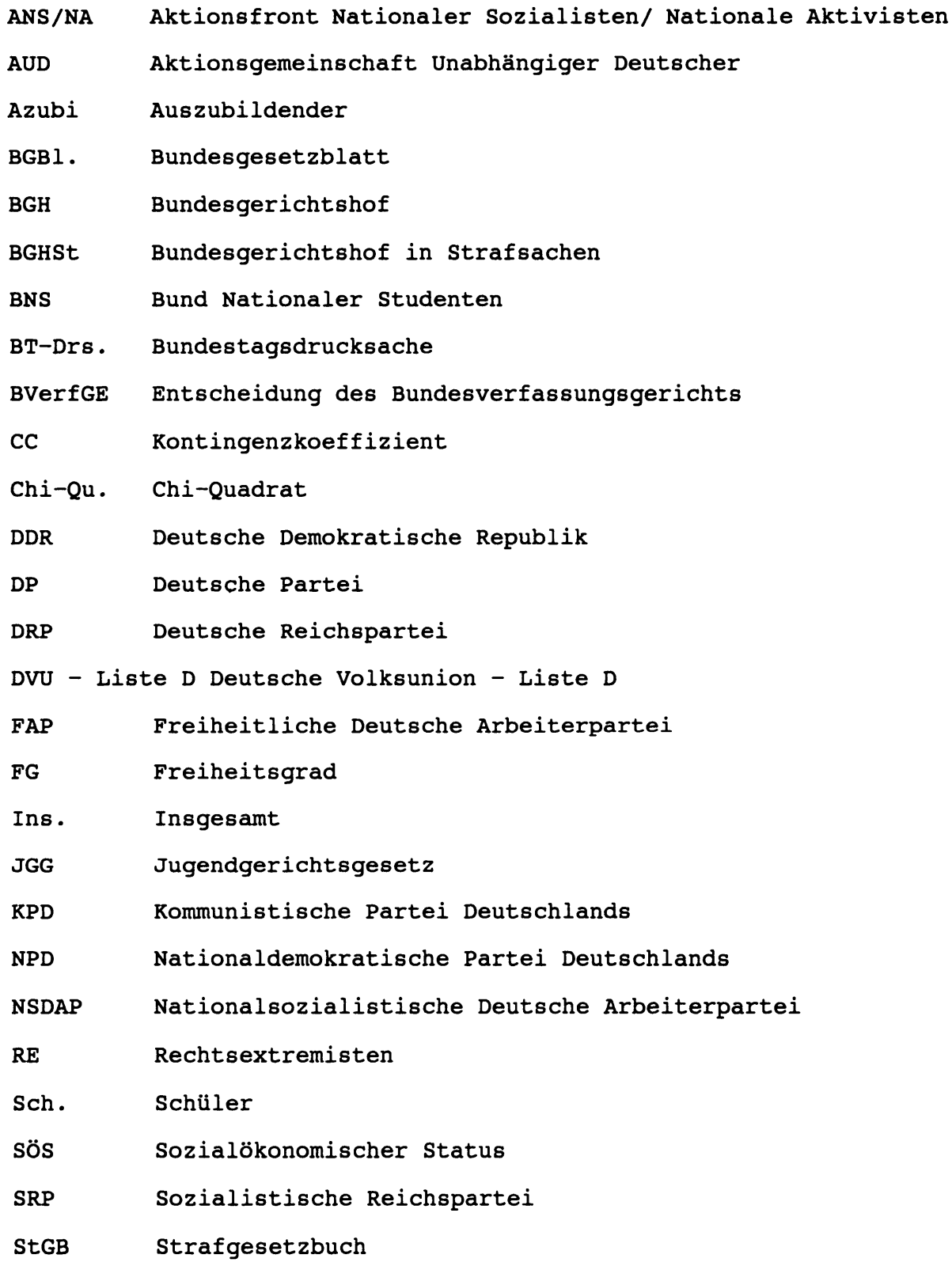

